\title{
Fibroblast Growth Factor 9 Suppresses Striatal Cell Death Dominantly Through ERK Signaling in Huntington's Disease
}

\author{
Issa Olakunle Yusufa,b,c Pei-Hsun Cheng ${ }^{c}$ Hsiu-Mei Chen ${ }^{c}$ Yu-Fan Chang ${ }^{c}$ \\ Chih-Yi Changc Han-In Yang Chia-Wei Lin ${ }^{c}$ Shaw-Jenq Tsaic,d Jih-Ing Chuangr,d \\ Chia-Ching Wu ${ }^{d, e}$ Bu-Miin Huang ${ }^{d, e}$ H. Sunny Sun ${ }^{d, f}$ Shang-Hsun Yang ${ }^{a, c, d}$ \\ aTaiwan International Graduate Program in Interdisciplinary Neuroscience, National Cheng Kung \\ University and Academia Sinica, Taipei, 'DInstitute of Clinical Medicine, 'Department of Physiology, \\ 'Institute of Basic Medical Sciences, e'Department of Cell Biology and Anatomy, Institute of Molecular \\ Medicine, College of Medicine, National Cheng Kung University, Tainan Taiwan
}

\section{Key Words}

Fibroblast Growth Factor 9 - Striatal cell death - Cell signaling - Glial cell line-derived neurotrophic factor $\cdot$ Huntington's disease

\begin{abstract}
Background/Aims: Huntington's disease (HD) is a heritable neurodegenerative disorder, and there is no cure for HD to date. A type of fibroblast growth factor (FGF), FGF9, has been reported to play prosurvival roles in other neurodegenerative diseases, such as Parkinson's disease and Alzheimer's disease. However, the effects of FGF9 on HD is still unknown. With many similarities in the cellular and pathological mechanisms that eventually cause cell death in neurodegenerative diseases, we hypothesize that FGF9 might provide neuroprotective functions in HD. Methods: In this study, STHdh ${ }^{07 / Q^{7}}$ (WT) and STHdh ${ }^{\text {Q111/Q111 }}$ (HD) striatal knockin cell lines were used to evaluate the neuroprotective effects of FGF9. Cell proliferation, cell death and neuroprotective markers were determined via the MTT assay, propidium iodide staining and Western blotting, respectively. The signaling pathways regulated by FGF9 were demonstrated using Western blotting. Additionally, HD transgenic mouse models were used to further confirm the neuroprotective effects of FGF9 via ELISA, Western blotting and immunostaining. Results: Results show that FGF9 not only enhances cell proliferation, but also alleviates cell death as cells under starvation stress. In addition, FGF9 significantly upregulates glial cell line-derived neurotrophic factor (GDNF) and an anti-apoptotic marker, Bcl-xL, and decreases the expression level of an apoptotic marker, cleaved caspase 3. Furthermore, FGF9 functions through ERK, AKT and JNK pathways. Especially, ERK pathway plays a critical role to influence the effects of FGF9 toward cell survival and GDNF production. Conclusions: These results not only show the neuroprotective effects of FGF9, but also clarify the critical mechanisms in HD cells, further providing an insight for the therapeutic potential of FGF9 in HD.

I. O. Yusuf, P.-H. Cheng and H.-M. Chen contributed equally to this paper.




\section{Introduction}

Fibroblast growth factors (FGFs) are important growth factors for physiological systems, and play critical and multifunctional roles during development, tumorigenesis, neuronal systems and disease progressions [1-6]. FGF9, originally discovered from a human glioma cell line, is one member of FGF family, and displays similar structure of heparin binding polypeptides with other FGF members [7-9]. In brain, FGF9 differentially expresses and highly influences the functional development in different brain regions [10-12], suggesting FGF9 signaling plays an important role for brain development and functions.

FGF9 has been reported to involve in neuronal disorders $[13,14]$. In neurodegenerative diseases, FGF9 signaling has been reported to affect cellular or pathological features in Alzheimer's disease (AD) and Parkinson's disease (PD) [5, 15-17]. For example, FGF9 is surrounding senile plaques in AD brain $[15,18]$. In PD models, FGF9 provide neuronprotective effects from apoptosis through $\gamma$-glutamylcysteine synthetase and heme oxygenase 1 expression $[5,16]$. These suggest the involvement of FGF9 signaling in neuropathology and disease progression of AD and PD. Since neurodegenerative diseases share certain similar characteristics, it strongly suggests FGF9 pathway might also play an important role in other neurodegenerative diseases, such as Huntington's disease (HD).

$\mathrm{HD}$ is one of neurodegenerative diseases, and is caused by the expansion of CAG repeats in the exon 1 region of Huntingtin(HTT) gene [19]. The mutant and misfolded HTT results in the formation of oligomers, and finally develops to pathological characteristics, including nuclear, intranuclear and neuropil aggregates [20]. The symptoms of this disease include motor and cognitive dysfunctions, such as emotional disturbances, mental deterioration, chorea and dystonia $[20,21]$. To date, there is no cure for this disease; as a result, those patients suffer unimaginable pain till death.

FGF signaling has been reported to involve in the pathogenesis of HD [22-26]. Especially, FGF2 is most studied among members of FGF family, and it shows the functions of enhancing neurogenesis and survival rate in striatal regions [24, 26]. Most importantly, FGF2 signaling not only improves neuropathological characteristics, but also alleviates longevity in HD transgenic mice [26]. These results suggest the important role of FGF signaling in HD. Since FGF family shares several similar signaling pathways and there is no study addressing on the effects of FGF9 on HD, we hypothesize FGF9 might also provide neuroprotective functions in HD. In this study, we used HD striatal cell lines to investigate the effects of FGF9, and showed the anti-apoptotic functions dominantly through the specific signaling pathway.

\section{Materials and Methods}

Cells and treatments

$\mathrm{STHdh}^{\mathrm{Q7/Q7}}(\mathrm{Q} 7)$ and $\mathrm{STHdh}^{\mathrm{Q111} / \mathrm{Q111}^{11}(\mathrm{Q} 111)}$ striatal cells expressing full-length wild type HTT with 7 CAG (Q) repeats (homozygous) and full length mutant HTT with $111 \mathrm{Q}$ (homozygous), respectively, were used in this study [27]. Cells were cultured in Dulbecco's modified Eagle's medium (DMEM) (Gibco) supplemented with $10 \%$ (v/v) fetal bovine serum (FBS, Hyclone), L-glutamine (2 mM), penicillin (100 U/mL, Gibco), streptomycin $(100 \mathrm{U} / \mathrm{mL}$, Gibco) and G418 (350 $\mu \mathrm{g} / \mathrm{mL}, \mathrm{MDBio})$ at $33^{\circ} \mathrm{C}$ in humidified condition and $5 \% \mathrm{CO}_{2}$. To treat cells with FGF9, cells were starved overnight using the medium with $1 \%(\mathrm{v} / \mathrm{v}) \mathrm{FBS}$, added FGF9 (R \& D Systems) and then cultured for 48 hours. To treat U0126 (Promega), cells were pretreated with U0126 $(10 \mu \mathrm{M}) 30$ minutes before FGF9 treatments, and then cultured for 48 hours. Cells were fixed or collected for the further examinations.

\section{Western blotting}

Cell and brain tissue samples were lysed using a sonicator (Qsonica), and then subjected to Sodium dodecyl sulfate polyacrylamide gel electrophoresis (Bio-Rad). Separated proteins were transferred onto PVDF membranes (Bio-Rad), and then hybridized with primary 
antibodies, including pAKT (Abcam; 1:1,000 dilution), tAKT(Abcam; 1:4,000 dilution), pJNK (Cell Signaling; 1:1,000 dilution), tJNK (Cell Signaling; 1:2,000 dilution), pERK (Cell Signaling; 1:1,000 dilution), tERK (Cell Signaling; 1:2,000 dilution), GDNF (Abcam; 1:1000 dilution), BcL-xL (Cell Signaling; 1:1,000 dilution), Cleaved caspase-3 (Cell Signaling; 1:500 dilution), FGF9 (Abcam; 1: 1,000 dilution) and $\gamma$-tubulin (Sigma; 1:10,000 dilution) antibodies. Protein expression levels were determined by an Amersham ECL kit (PerkinElmer) through an imaging system (ChampGel), and expression profiling was quantitated by ImageJ system $(\mathrm{NIH})$.

\section{Propidium iodide (PI) staining}

Q7 and Q111 cells were seeded in 96-well plates with different treatments, and then subjected for PI staining to determine cell apoptosis 48 hours after treatments. $50 \mu \mathrm{L}$ of PI $(1 \mu \mathrm{g} / \mathrm{mL}$, Sigma) was added to each well, and incubated for 15 minutes in the dark at room temperature. Medium was gently removed, and Hoechst $33342(1 \mu \mathrm{g} / \mathrm{mL}$, Sigma) was added to the cells to stain the nucleus. These cells were then incubated in the dark at room temperature for 20 minutes, and fluorescence images were captured using a DMi8 fluorescent microscope (Leica) with a Metamorph software. ImageJ system (NIH) was used to count PI positive cells and nuclear Hoechst signals.

\section{Enzyme-linked immunosorbent assay (ELISA)}

Brain tissues were lysed using a sonicator (Qsonica), and then centrifuged to obtain the supernatants. The protein concentration of supernatants were determined by Bradford method, and equal amounts of protein were subjected to a FGF9 enzyme-linked immunosorbent assay (R\&D Systems) according to manufacturer's protocol. The FGF9 concentration was detected by an ELISA reader.

MTT Assay

Q7 and Q111 cells were seeded in 96-well plates with different treatments as described above. Cell proliferation was assessed by adding $50 \mu \mathrm{L}$ of 3-(4,5-dimethylthiazolyl-2)-2,5diphenyltetrazolium bromide (MTT, Calbiochem, $0.5 \mathrm{mg} / \mathrm{mL}$ ) into each well, and then incubated in the dark at $33^{\circ} \mathrm{C}$ for 4 hours. After incubation, medium and MTT reagent were gently removed and 50 $\mu \mathrm{L}$ Dimethyl sulfoxide (DMSO, Amresco) was added to each well to solubilize the MTT-formazan crystals. The plates were shaken for 20 minutes at room temperature, and subjected for an ELISA reader.

Transgenic mice

The transgenic mice used in this study include R6/2 [28] and Ubi-G-HTT84Q transgenic mice [29]. The animal protocol was approved by the Institutional Animal Care and Use Committee at National Cheng Kung University, Taiwan. HD R6/2 mice carry truncated exon1 with expanded polyQ under control of a HTT promoter. Ubi-G-HTT84Q transgenic mice carry the expanded polyQ in truncated exon 1 of HTT gene fused with green fluorescence protein (GFP) gene under control of a human ubiquitin promoter.

\section{Statistical Analyses}

Data are presented as mean \pm standard error of mean (SEM). Student's t-test was used to compare differences between two different groups, and one-way analysis of variance (ANOVA) for more than two different groups using commercial statistical software (GraphPad Prism 4.02). Tukey's post-hoc test was used to test differences among different groups. Statistical significance was set at $\mathrm{P}<0.05$.

\section{Results}

To understand the effects of FGF9 on HD, we used STHdh ${ }^{\text {Q7/Q7 }}$ (Q7; normal control) and STHdh ${ }^{\mathrm{Q111} / \mathrm{Q} 111}$ (Q111; HD model) striatal cells derived from wild-type and Hdh(Q111) 
knock-in mice, respectively, as our models [27]. Since FGF9 is an important growth factor for development and tumorigenesis [1-6], we first examine whether FGF9 could stimulate cell proliferation in these two cell lines. We treated cells with different concentration of FGF9 (50 ng/mL and $100 \mathrm{ng} / \mathrm{mL}$ ), cultured for 48 hours, and then subjected to the 3-(4,5-Dimethylthiazol-2-yl)-2,5-diphenyltetrazolium bromide (MTT) assay. As shown in Fig. 1A, the higher density of Q7 and Q111 cells was observed after the treatment of FGF9 compared to that of vehicle controls, and quantitation results showed either $50 \mathrm{ng} / \mathrm{mL}$ or $100 \mathrm{ng} / \mathrm{mL}$ of FGF9 could enhance cell proliferation (Fig. 1B), suggesting FGF9 could play a role of growth factor in HD striatal cell lines.

The Q111 cells have been reported to be vulnerable to cell death under exogenous stress [30]. Therefore, we are curious about whether FGF9 could diminish cell death of these striatal cell lines under exogenous stress. We cultured Q7 and Q111 cells with 1\% fetal bovine serum (FBS), and examined cell death with or without FGF9 treatments. After 2-day culture under starvation condition, we observed the signals of cell death using Propidium iodide (PI) staining in these two cell lines (Fig. 2A). As we treated cells with FGF9, the cell death rate was significantly decreased in Q7 and Q111 cells (Fig. 2A and 2B). In addition,

Fig. 1. FGF9 increases cell proliferation in Q7 and Q111 cells. Q7 and Q111 cells were treated with different concentration of FGF9 for 48 hours, and subjected for a MTT assay. (A) Images show the cell morphology of Q7 (top panel) and Q111 (bottom panel) cells under transmission light. (B) MTT assays show the significant increase of cell proliferation after FGF9 treatments in two cell lines. * represents $\mathrm{P}<0.05$. $\mathrm{N}=3$.

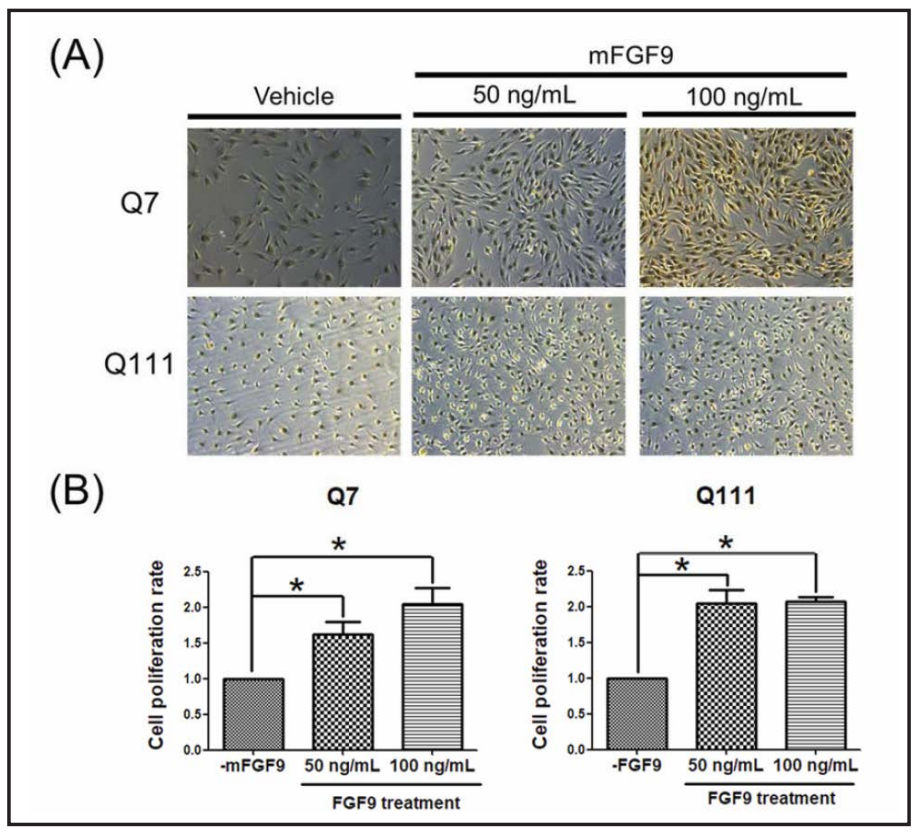

Fig. 2. FGF9 alleviates cell death in Q7 and Q111 cells. Q7 and Q111 cells were treated with FGF9 (50 ng/mL) for 48 hours, and subjected for the PI staining. (A) Fluorescence images show the death of cells (red) with or without FGF9 treatments. Blue color represents Hoechst staining. (B) Quantitation results show the suppression of cell death rate after FGF9 treatments in Q7 and Q111 cells. * represents $\mathrm{P}<0.05$. $\mathrm{N}=6$.

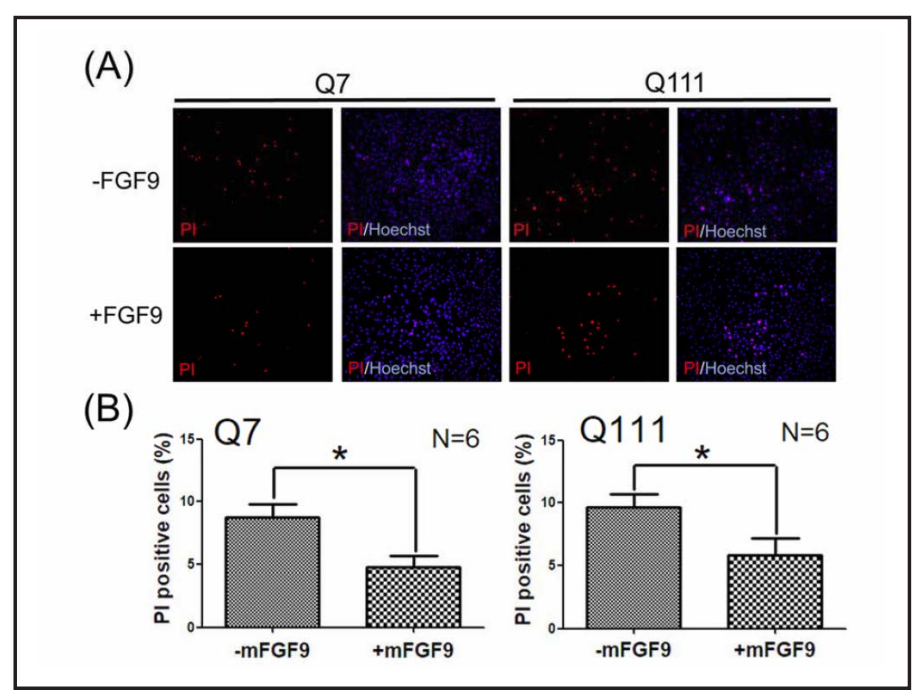


Fig. 3. FGF9 decreases the expression level of c-Cas3 and increases the expression level of Bcl-xL in Q7 and Q111 cells. Q7 and Q111 cells were treated with FGF9 (50 $\mathrm{ng} / \mathrm{mL}$ ) for 48 hours, and subjected for Western blotting. (A) Western blotting was performed using c-Cas3 and Bcl-xL antibodies. $\gamma$-tubulin

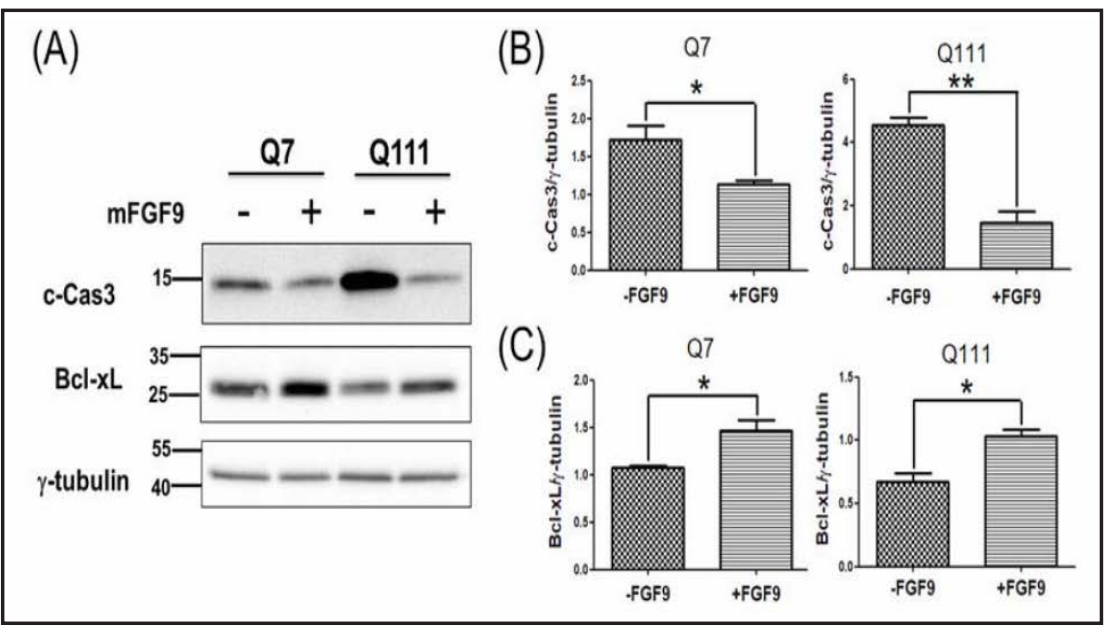
was used as an internal control. (B) Quantitation results show the suppression of c-Cas3 after FGF9 treatments in Q7 and Q111 cells. (C) Quantitation results show the increase of Bcl-xL after FGF9 treatments in Q7 and Q111 cells. * represents $\mathrm{P}<0.05$. ** represents $\mathrm{P}<0.01$. $\mathrm{N}=3$.

we also extracted cell protein from these cells, and determined the expression of cleaved caspase 3 (c-Cas3, an apoptosis marker) and B-cell lymphoma-extra large (Bcl-xL, an anti-apoptosis marker) proteins. Since we observed less PI-positive signal as we treated cells with FGF9 (Fig. 2), we also detected lower expression level of c-Cas3 and higher expression level of Bcl-xL in FGF9 treated cells (Fig. 3A). Quantitated results from these Western blotting showed that FGF9 could significantly suppress the c-Cas 3 level (Fig. 3B) and increase Bcl-xL level (Fig. 3C). These suggest FGF9 not only increases cell proliferation, but also provides certain unknown functions against cell death.

Glial cell-derived neurotrophic factor (GDNF) has been reported to be functional against neurotoxicity in HD Q111 cells [31]. Since we observed FGF9 suppresses the cell

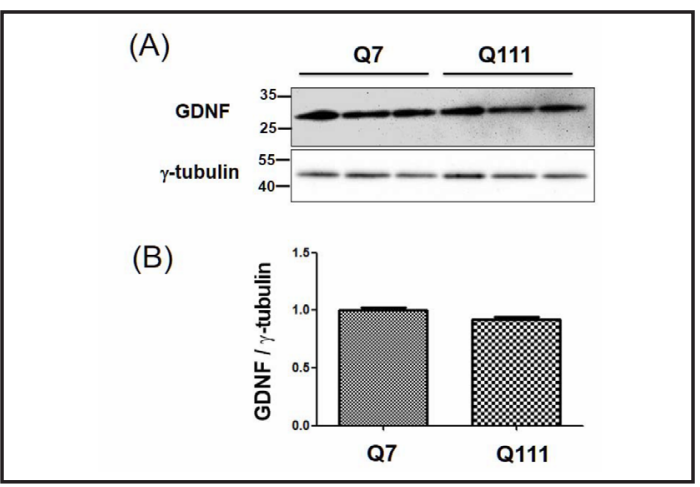

Fig. 4. Similar expression levels of endogenous GDNF in Q7 and Q111 cells. Q7 and Q111 cells were cultured for 48 hours, and subjected for Western blotting. (A) Western blotting was performed using a GDNF antibody. $\gamma$-tubulin was used as an internal control. (B) Quantitation results show there is no difference of GDNF expression in Q7 cells and Q111 cells. $\mathrm{N}=3$. death in these HD cells, we further determine the expression level of GDNF after the FGF9 treatment. We first compared the expression of endogenous GDNF in Q7 and Q111 cells without treatments, showing similar expression level of GDNF (Fig. 4). In Q7 cells, higher trend of GDNF expression was detected in FGF9 treated cells, but there was no significant difference (Fig. 5A and 5C). However, the expression level of GDNF was significantly increased in Q111 cells after FGF9 treatment (Fig. 5B and 5D), suggesting FGF9 enhances cell survival through increased level of GDNF in HD cells.

FGF9 has physiological functions through several down-stream pathways, such as Protein Kinase B (AKT), extracellular signal-regulated kinases (ERK) and c-Jun N-terminal kinases (JNK) signals [32]. In order to identify the critical pathways for beneficial effects of FGF9 in HD, we examine the activation profiling of AKT, ERK and JNK pathways after the treatment of FGF9. We collected cell samples at $0,15,30$ and 60 minutes after FGF9 treatment, and subjected samples for Western blotting using specific antibodies. In AKT signaling, phosphorylated AKT was significantly increased in Q7 (Fig. 6A) and Q111 cells (Fig. 
6B) after FGF9 treatments for 15 minutes, and the phosphorylated status was maintained for 30 minutes and significantly decreased after 60 minutes in both cell lines (Fig. 6C and 6D). In ERK signaling, the increased trend of phosphorylated ERK was observed in Q7 cells after treatment of FGF9, but it did not reach significant difference after the statistical analyses (Fig. 7A and 7C). However, in Q111 cells, the increase of phosphorylated ERK was significantly and highly displayed at 15 minutes and maintained for 30 minutes after the treatments of FGF9 (Fig. 7B and 7D). As to JNK signaling, FGF9 could significantly activate the expression of phosphorylated JNK after 15-minute treatment in Q7 (Fig. 8A and 8C) and Q111 (Fig. 8B and $8 \mathrm{D}$ ) cells, but the signaling was decreased soon after 30-minute treatment. These results suggest FGF9 activates AKT, ERK and JNK pathways to enhance cell survival in HD cell lines.

Based on the above study of signal transduction, we found the activation of ERK signaling is the most significant one (Fig. 7). As a result, we speculate the ERK signaling is the dominant pathway to enhance the expression of GDNF, further facilitating cell survival in HD cells. Using U0126 (an ERK inhibitor), we tried to block the ERK pathway and examine cell survival rate via the PI staining. As shown in Fig. 9A, FGF9 decreased the signal of PI staining in Q7 and Q111 cells, whereas U0126 reversed this protective effect to lead to more death signal. Quantitated results also showed U0126 significantly blocked the anti-death function

Fig. 5. FGF9 increases the expression level of GDNF in Q7 and Q111 cells. Q7 and Q111 cells were treated with FGF9 (50 ng/ $\mathrm{mL}$ ) for 48 hours, and subjected for Western blotting. (A and B) Western blotting was performed using a GDNF antibody in Q7 (A) and Q111(B) cells. $\gamma$ - tubulin was used as an internal control. (C and D) Quantitation results show the increased trend of GDNF expression in Q7 cells(C) and significant increase of GDNF in Q111 cells (D) after FGF9 treatments. * represents $\mathrm{P}<0.05 . \mathrm{N}=3$.
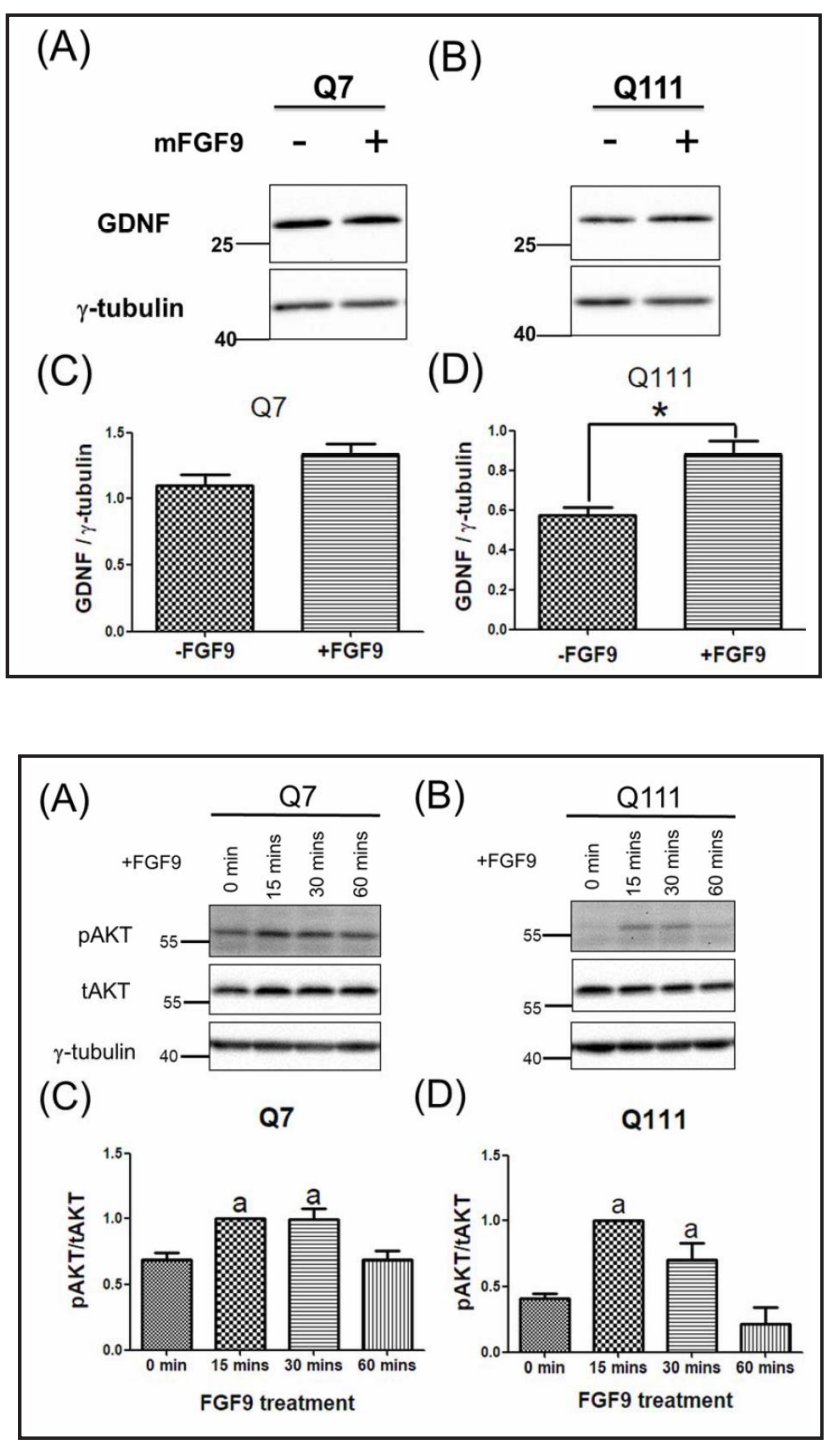

Fig. 6. FGF9 activates AKT signaling in Q7 and Q111 cells. Q7 and Q111 cells were treated with FGF9 (50 ng/mL) for $0,15,30$ and 60 minutes, and subjected for Western blotting. (A and B) Western blotting was performed using pAKT and tAKT antibodies in Q7(A) and Q111(B) cells. $\gamma$ - tubulin was used as an internal control. (C and D) Quantitation results show the activation of pAKT after normalization by tAKT in Q7 cells(C) and Q111 cells (D) after FGF9 treatments. Different characters on different bars indicate statistically significant difference $(\mathrm{P}<0.05) . \mathrm{N}=3$. 
of FGF9 in both cell lines (Fig. 9B and 9C). We further confirmed the expression level of anti-apoptosis marker, Bcl-xL, and found U0126 did suppress the expression level of Bcl-xL induced by FGF9 in both cell lines (Fig. 10A and 10B). In addition, since we confirmed FGF9 could increase the expression of GDNF (Fig. 5), we also examined the role of ERK signaling in the production of GDNF. In Q7 cells, slight decrease of GDNF expression was observed

Fig. 7. FGF9 activates ERK signaling in Q111 cells. Q7 and Q111 cells were treated with FGF9 (50 ng/mL) for 0, 15, 30 and 60 minutes, and subjected for Western blotting. (A and B) Western blotting was performed using pERK and tERK antibodies in Q7(A) and Q111(B) cells. $\gamma$ - tubulin was used as an internal control. (C and D) Quantitation results show the activation of pERK after normalization by tERK in Q111 cells (D), but not in Q7 cells(C) after FGF9 treatments. Different characters on different bars indicate statistically significant difference $(\mathrm{P}<0.05)$. $\mathrm{N}=3$.

Fig. 8. FGF9 activates JNK signaling in Q7 and Q111 cells. Q7 and Q111 cells were treated with FGF9 (50 ng/mL) for $0,15,30$ and 60 minutes, and subjected for Western blotting. (A and B) Western blotting was performed using pJNK and tJNK antibodies in Q7 (A) and Q111 (B) cells. $\gamma$ - tubulin was used as an internal control. (C and D) Quantitation results show the activation of pJNK after normalization by tJNK in Q7 cells(C) and Q111 cells (D) after FGF9 treatments. Different characters on different bars indicate statistically significant difference $(\mathrm{P}<0.05) . \mathrm{N}=3$.

Fig. 9. FGF9 alleviates cell death through the ERK signaling in Q7 and Q111 cells. Q7 and Q111 cells were pretreated with U0126 (U) and then treated with FGF9 (50 $\mathrm{ng} / \mathrm{mL}$ ) for 48 hours. Cells were fixed and subjected for the PI staining. (A) Fluorescence images show the death of cells (red) after different treatments as indicated. Blue color represents Hoechst staining. (B and C) Quantitation results show the blockage of FGF9 effects on cell survival after the U0126 treatment in Q7 (B) and Q111(C) cells. * represents $\mathrm{P}<0.05$. $\mathrm{N}=8$.
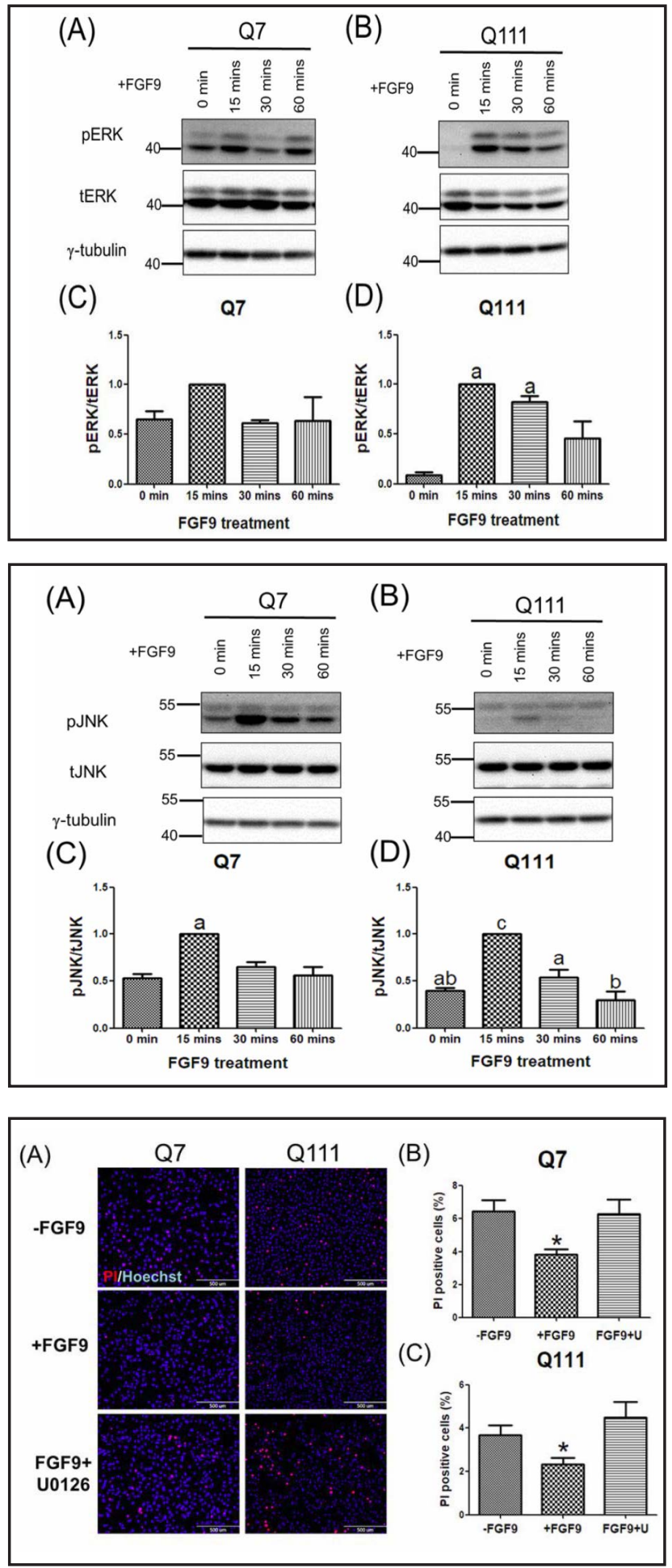
Fig. 10. FGF9 alters expression levels of Bcl-xL and GDNF through the ERK signaling in Q7 and Q111 cells. Q7 and Q111 cells were pretreated with U0126 (U) and then treated with FGF9 (50 ng/mL) for 48 hours. Cell samples were subjected for the Western blotting. (A) Western blotting was performed using Bcl-xL and GDNF antibodies. $\gamma$-tubulin was used as an internal control. (B and C) Quantitation results show the blockage of FGF9 effects on increase of

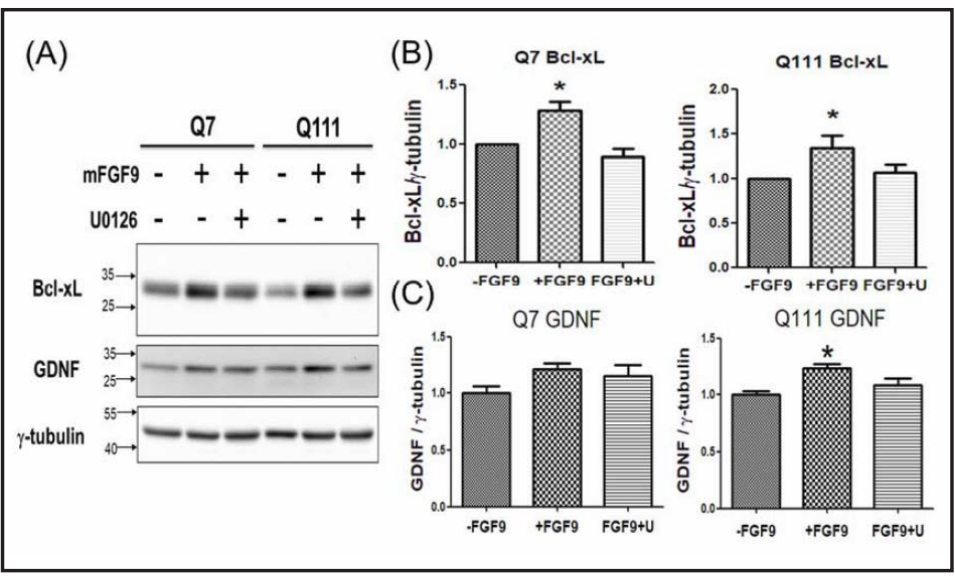
Bcl-XL (B) and increase of GDNF (C) after the U0126 treatment in Q7 and Q111 cells. * represents P<0.05. $\mathrm{N}=8-10$.

after the treatment of U0126 (Fig. 10A and 10C); however, U0126 did also significantly suppress the expression of GDNF in Q111 cells. Combining these results, it shows FGF9 dominantly provides anti-apoptotic functions through the ERK signaling.

Last, we are curious about the FGF9 expression profiling in vivo. Two transgenic mouse models were used in this study, including R6/2 [28] and Ubi-G-HTT84Q transgenic mice [29]. First, the cortex samples of R6/2 and wild-type mice were collected at 3.5 months of age (end stage for R6/2 transgenic mice), and subjected to Western blotting. As shown in Fig. 11A and 11B, lower expression level of FGF9 was displayed in the brains of R6/2 transgenic mice. In Ubi-GHTT84Q transgenic mice before the onset, lower expression level of FGF9 was also detected in brains of Ubi-G-HTT84Q mice using enzyme-linked immunosorbent assay (ELISA) compared to that of Ubi-G-HTT19Q control mice (Fig. 12). These results suggest the deficit of FGF9 expression in brains of HD transgenic mice in vivo.

\section{Discussion}

HD is a neurodegenerative disease, and is still without any absolute remedy; therefore, it is necessitating huge research efforts into developing a therapeutic solution. In this study, we show that FGF9 enhances cell proliferation, increases cell survival, suppresses caspase-3 activation and upregulates Bcl-xL in HD striatal cells. In

(A)

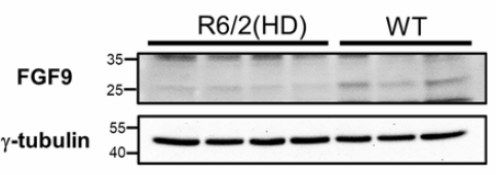

(B)

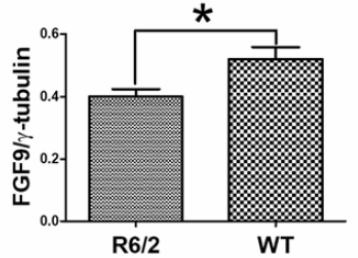

Fig. 11. Lower expression level of FGF9 in R6/2 HD transgenic mice. Cortex samples from R6/2 HD transgenic mice were subjected to Western blotting. (A) Western blotting using cortex samples from R6/2 HD transgenic and wild-type (WT) mice at 3.5 months of age shows lower expression level of FGF9 in R6/2 HD transgenic mice. (B) Quantitation results show the expression of endogenous FGF9 is lower in R6/2 HD transgenic mice. * represents $\mathrm{P}<0.05$.

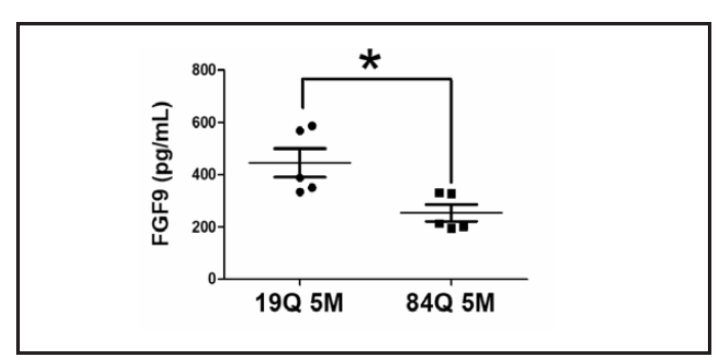

Fig. 12. Lower expression level of FGF9 in Ubi-G-HTT84Q HD transgenic mice. Cortex samples from UbiG-HTT84Q HD transgenic mice at 5 months of age were subjected to an ELISA assay, showing the lower expression level of FGF9 in Ubi-G-HTT84Q mice compared to that of Ubi-G-HTT19Q control mice at 5 months of age. * represents $\mathrm{P}<0.05$. 


\section{Cellular Physiology Cell Physiol Biochem 2018;48:605-617 \\ \begin{tabular}{l|l} 
DOI: 10.1159/000491889 & $\begin{array}{l}\text { O } 2018 \text { The Author(s). Published by S. Karger AG, Basel } \\
\text { www.karger.com/cpb }\end{array}$
\end{tabular} \\ Yusuf et al.: Neuroprotective Effects of FGF9 in Huntington's Disease}

Fig. 13. FGF9 increases the expression level of GDNF and decreases the TUNEL signals in R6/2 transgenic mice. Stereotaxical injection was performed using FGF9 lentiviruses in striatum of R6/2 HD transgenic mice at 2 months of age. Immunofluorescence staining shows the over-expression of FGF9 protein (Flag, Green, left panel), higher expression of GDNF (GDNF, Red) and lower signal of cell death (TUNEL, Green, right panel) after injection of FGF9 viruses compared to those of sham controls. Blue color represents Hoechst staining.

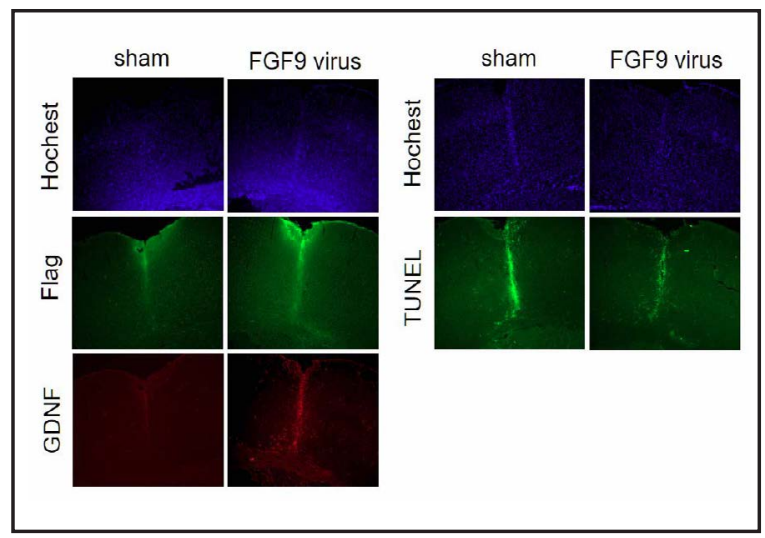

addition, we also show that FGF9 upregulates one neurotrophic factor, GDNF, and activates AKT, ERK and JNK signaling. Especially, we demonstrate that neuroprotective effect induced by FGF9 is dominantly mediated through ERK signaling. These results show not only one of FGF9 regulatory pathways for neuroprotection, but also an insight for the therapeutical strategy toward HD.

FGF signaling has been reported to involve in the pathogenesis of HD [22-26]. Especially, FGF2 is most studied among members of FGF family, and it shows the functions of enhancing neurogenesis and survival rate in striatal regions, further ameliorating neuropathological aggregates, motor symptoms and lifespan in R6/2 HD transgenic mice [24, 26]. Since FGF2 and FGF9 share several receptors, such as FGFR3, it highly suggests FGF2 and FGF9 may lead to similar protective effects. Indeed, in this study, we observed FGF9 increases cell proliferation and suppresses cell death in HD striatal cell lines (Fig. 1-3), suggesting FGF9 might also provide protective functions similar in FGF2 studies in vivo. Due to limited HD mice we have, we tried a preliminary study to stereotaxically inject FGF9 lentiviruses into striatum of HD transgenic mice, showing FGF9 could increase the expression level of GDNF and decrease cell death in HD mice (Fig. 13). We will further confirm these results, and also generate FGF9 transgenic mice to examine the effects of FGF9 in HD in vivo via genetic experiments.

Neurotrophic factors play a vital role in the development and maintenance of vertebrate nervous system, regulating neuronal survival, and synaptic functions and plasticity [33]. The withdrawal of neurotrophic support has been increasingly implicated in the pathogenesis of neurodegenerative diseases [33-35]. A particular neurotrophic factor, GDNF, has been linked with different neurodegenerative diseases, such as PD, AD and HD [36-38]. In our study, we observed FGF9 could significantly enhance the expression level of GDNF in Q111 cells, but not in Q7 cells, through the ERK signaling (Fig. 7 and 10). These suggest the specific role of FGF9 to generate GDNF under the HD condition. Since GDNF has been reported to ameliorate pathological and behavioral phenotypes in different models of HD [37, 39, 40], the specific functions of FGF9 to generate GDNF may be applied for potential therapy, even applying to other neuronal diseases.

MAPK-ERK, PI3-AKT and JNK signaling cascades are the most commonly reported pathways employed by FGFs [41]. Our data is attuned as we show that FGF9 significantly activates ERK, AKT and JNK predominantly peaking after 15 minutes of FGF9 treatment and subsequently declining in Q111 cells (Fig. 6, 7 and 8). These results are consistent with earlier reports [32, 42], indicating the activation of these pathways induced by FGF9 could be common mechanisms in different tissues. In addition, the phenomenon of rapid and short-lived activation followed by decline of these signaling pathways indicates that FGF9 could only transiently activate these pathways and is tightly regulated by the feedback mechanisms [41, 43-45]. Furthermore, different responses to FGF9 were observed between Q7 and Q111 cells, such as the phosphorylation of ERK (Fig. 7), suggesting Q111 cells under the HD condition may have different regulatory responses against disease progression. 
Therefore, long-term effects, side effects and disease-specific phenotypes from FGF9 should be longitudinally examined.

In previous reports, dysregulation of ERK signaling is observed in HD, and activation of ERK pathway provides beneficial function in HD [46]. In our result, we found the activation of ERK signaling is the most significant response after the FGF9 treatment (Fig. 7), and suppression of this ERK pathway could abolish the protective effects (Fig. 9 and 10). These support the neuroprotective role of FGF9 in HD through ERK. In addition, ERK signaling is required for the expression of GDNF in spermatogonial stem cells [47]. In our study, we also observed suppression of the ERK pathway inhibited the expression level of GDNF in Q111 cells, but not in Q7 cells (Fig. 10), suggesting ERK signaling is also critical for the production of GDNF in the HD cells. Again, since GDNF has been reported to provide beneficial effects on HD [40] and other neurodegenerative diseases [48], FGF9 might also be able to provide neuroprotective functions through ERK signaling in other neuronal diseases.

Based on the knowledge we have, there is no study focusing on FGF9 and HD till now although there are some reports or database indicating abnormal expression of FGF9 in HD $[49,50]$. In this study, we showed FGF9 could provide anti-apoptosis functions through ERK, AKT and JNK pathways in HD, and ERK signaling is a critical regulatory mechanism to affect the expression of GDNF and resulting cell survival rate. Since this is a proof of concept study and preliminary data in vivo showing FGF9 could suppress the cell apoptosis in HD transgenic mice, we will further use genetic methods to examine the effects of FGF9 on HD in vivo. With the achievement of this study, we wish to provide an insight for the potential therapy in HD.

\section{Authors and Contributors}

IOY, PHC, HMC, YFC, CYC, HIY, CWL, JIC and SHY handled animal studies, molecular analysis and analyzed data; SJT, JIC, CCW, BMH, HSS and SHY designed the experiments and oversaw the progression of this study. IOY, HMC and SHY drafted the paper. All authors read and approved the final manuscript.

\section{Acknowledgements}

This work was supported by the Ministry of Science and Technology (MOST 105-2628-B-006-015-MY3, MOST 105-2320-B-006-004 and MOST 106-2320-B-006-004) and, in part, the Ministry of Education, Taiwan, R.O.C. The Aim for the Top University Project of National Cheng Kung University (NCKU).

\section{Disclosure Statement}

None declared.

\section{References}

1 Ohgino K, Soejima K, Yasuda H, Hayashi Y, Hamamoto J, Naoki K, Arai D, Ishioka K, Sato T, Terai H, Ikemura S, Yoda S, Tani T, Kuroda A, Betsuyaku T: Expression of fibroblast growth factor 9 is associated with poor prognosis in patients with resected non-small cell lung cancer. Lung Cancer 2013;83:90-96.

-2 Chung CL, Lu CW, Cheng YS, Lin CY, Sun HS, Lin YM: Association of aberrant expression of sex-determining gene fibroblast growth factor 9 with Sertoli cell-only syndrome. Fertil Steril 2013;100:1547-1554. 


\section{Cellular Physiology Cell Physiol Biochem 2018;48:605-617 and Biochemistry Publisher \begin{tabular}{l|l} 
DOI: 10.1159/000491889 & $\begin{array}{l}\text { (c) } 2018 \text { The Author(s). Published by S. Karger AG, Basel } \\
\text { www.karger.com/cpb }\end{array}$
\end{tabular}}

Yusuf et al.: Neuroprotective Effects of FGF9 in Huntington's Disease

3 Lin SC, Wang CC, Wu MH, Yang SH, Li YH, Tsai SJ: Hypoxia-induced microRNA-20a expression increases ERK phosphorylation and angiogenic gene expression in endometriotic stromal cells. J Clin Endocrinol Metab 2012;97:E1515-1523.

4 Frontini MJ, Nong Z, Gros R, Drangova M, O'Neil C, Rahman MN, Akawi O, Yin H, Ellis CG, Pickering JG: Fibroblast growth factor 9 delivery during angiogenesis produces durable, vasoresponsive microvessels wrapped by smooth muscle cells. Nat Biotechnol 2011;29:421-427.

5 Huang JY, Chuang JI: Fibroblast growth factor 9 upregulates heme oxygenase-1 and gammaglutamylcysteine synthetase expression to protect neurons from 1-methyl-4-phenylpyridinium toxicity. Free Radic Biol Med 2010;49:1099-1108.

-6 Bowles J, Feng CW, Spiller C, Davidson TL, Jackson A, Koopman P: FGF9 suppresses meiosis and promotes male germ cell fate in mice. Dev Cell 2010;19:440-449.

7 Naruo K, Seko C, Kuroshima K, Matsutani E, Sasada R, Kondo T, Kurokawa T: Novel secretory heparinbinding factors from human glioma cells (glia-activating factors) involved in glial cell growth. Purification and biological properties. J Biol Chem 1993;268:2857-2864.

8 Behr B, Leucht P, Longaker MT, Quarto N: Fgf-9 is required for angiogenesis and osteogenesis in long bone repair. Proc Natl Acad Sci U S A 2010;107:11853-11858.

9 Hecht D, Zimmerman N, Bedford M, Avivi A, Yayon A: Identification of fibroblast growth factor 9 (FGF9) as a high affinity, heparin dependent ligand for FGF receptors 3 and 2 but not for FGF receptors 1 and 4. Growth Factors 1995;12:223-233.

10 Lum M, Turbic A, Mitrovic B, Turnley AM: Fibroblast growth factor-9 inhibits astrocyte differentiation of adult mouse neural progenitor cells. J Neurosci Res 2009;87:2201-2210.

11 Lin Y, Chen L, Lin C, Luo Y, Tsai RY, Wang F: Neuron-derived FGF9 is essential for scaffold formation of Bergmann radial fibers and migration of granule neurons in the cerebellum. Dev Biol 2009;329:44-54.

12 Tagashira S, Ozaki K, Ohta M, Itoh N: Localization of fibroblast growth factor-9 mRNA in the rat brain. Brain Res Mol Brain Res 1995;30:233-241.

13 Garrett L, Becker L, Rozman J, Puk O, Stoeger T, Yildirim AO, Bohla A, Eickelberg O, Hans W, Prehn C, Adamski J, Klopstock T, Racz I, Zimmer A, Klingenspor M, Fuchs H, Gailus-Durner V, Wurst W, Hrabe de Angelis M, Graw J, Holter SM: Fgf9 Y162C Mutation Alters Information Processing and Social Memory in Mice. Mol Neurobiol 2018; 55:4580-4595.

14 Turner CA, Eren-Kocak E, Inui EG, Watson SJ, Akil H: Dysregulated fibroblast growth factor (FGF) signaling in neurological and psychiatric disorders. Semin Cell Dev Biol 2016;53:136-143.

15 Nakamura S, Arima K, Haga S, Aizawa T, Motoi Y, Otsuka M, Ueki A, Ikeda K: Fibroblast growth factor (FGF)9 immunoreactivity in senile plaques. Brain Res 1998;814:222-225.

16 Huang JY, Hong YT, Chuang JI: Fibroblast growth factor 9 prevents MPP+-induced death of dopaminergic neurons and is involved in melatonin neuroprotection in vivo and in vitro. J Neurochem 2009;109:14001412.

17 Johanson C, McMillan P, Tavares R, Spangenberger A, Duncan J, Silverberg G, Stopa E: Homeostatic capabilities of the choroid plexus epithelium in Alzheimer's disease. Cerebrospinal Fluid Res 2004;1:3.

18 Takami K, Matsuo A, Terai K, Walker DG, McGeer EG, McGeer PL: Fibroblast growth factor receptor-1 expression in the cortex and hippocampus in Alzheimer's disease. Brain Res 1998;802:89-97.

19 Group THsDCR: A novel gene containing a trinucleotide repeat that is expanded and unstable on Huntington's disease chromosomes. Cell 1993;72:971-983.

20 Yang SH, Chan AW: Transgenic Animal Models of Huntington's Disease. Curr Top Behav Neurosci 2011;7:61-85.

21 Moumne L, Betuing S, Caboche J: Multiple Aspects of Gene Dysregulation in Huntington's Disease. Front Neurol 2013;4:127.

22 Moraes L, Vasconcelos-dos-Santos A, Santana FC, Godoy MA, Rosado-de-Castro PH, Jasmin, AzevedoPereira RL, Cintra WM, Gasparetto EL, Santiago MF, Mendez-Otero R: Neuroprotective effects and magnetic resonance imaging of mesenchymal stem cells labeled with SPION in a rat model of Huntington's disease. Stem Cell Res 2012;9:143-155.

23 Rudenko O, Tkach V, Berezin V, Bock E: Effects of FGF receptor peptide agonists on animal behavior under normal and pathological conditions. Neurosci Res 2010;68:35-43. 


\section{Cellular Physiology Cell Physiol Biochem 2018;48:605-617 \begin{tabular}{ll|l} 
and Biochemistry Published online: July 18, 2018 & $\begin{array}{l}\text { (c) } 2018 \text { The Author(s). Published by S. Karger AG, Basel } \\
\text { www.karger.com/cpb }\end{array}$
\end{tabular}}

-24 Peng J, Xie L, Jin K, Greenberg DA, Andersen JK: Fibroblast growth factor 2 enhances striatal and nigral neurogenesis in the acute 1-methyl-4-phenyl-1,2,3,6-tetrahydropyridine model of Parkinson's disease. Neuroscience 2008;153:664-670.

25 La Spada AR: Huntington's disease and neurogenesis: FGF-2 to the rescue? Proc Natl Acad Sci U S A 2005;102:17889-17890.

-26 Jin K, LaFevre-Bernt M, Sun Y, Chen S, Gafni J, Crippen D, Logvinova A, Ross CA, Greenberg DA, Ellerby LM: FGF-2 promotes neurogenesis and neuroprotection and prolongs survival in a transgenic mouse model of Huntington's disease. Proc Natl Acad Sci U S A 2005;102:18189-18194.

27 Trettel F, Rigamonti D, Hilditch-Maguire P, Wheeler VC, Sharp AH, Persichetti F, Cattaneo E, MacDonald ME: Dominant phenotypes produced by the HD mutation in STHdh(Q111) striatal cells. Hum Mol Genet 2000;9:2799-2809.

28 Mangiarini L, Sathasivam K, Seller M, Cozens B, Harper A, Hetherington C, Lawton M, Trottier Y, Lehrach H, Davies SW, Bates GP: Exon 1 of the HD gene with an expanded CAG repeat is sufficient to cause a progressive neurological phenotype in transgenic mice. Cell 1996;87:493-506.

29 Cheng PH, Li CL, Her LS, Chang YF, Chan AW, Chen CM, Yang SH: Significantly differential diffusion of neuropathological aggregates in the brain of transgenic mice carrying $\mathrm{N}$-terminal mutant huntingtin fused with green fluorescent protein. Brain Struct Funct 2013;218:283-294.

-30 Ruan Q, Lesort M, MacDonald ME, Johnson GV: Striatal cells from mutant huntingtin knock-in mice are selectively vulnerable to mitochondrial complex II inhibitor-induced cell death through a non-apoptotic pathway. Hum Mol Genet 2004;13:669-681.

-31 Ruiz C, Casarejos MJ, Gomez A, Solano R, de Yebenes JG, Mena MA: Protection by glia-conditioned medium in a cell model of Huntington disease. PLoS Curr 2012;4:e4fbca54a2028b.

32 Lai MS, Cheng YS, Chen PR, Tsai SJ, Huang BM: Fibroblast growth factor 9 activates akt and MAPK pathways to stimulate steroidogenesis in mouse leydig cells. PloS one 2014;9:e90243.

-33 Dawbarn D, Allen SJ: Neurotrophins and neurodegeneration. Neuropathol Appl Neurobiol 2003;29,:211230.

34 Zuccato C, Cattaneo E: Brain-derived neurotrophic factor in neurodegenerative diseases. Nat Rev Neurol 2009;5:311-322.

-35 Ferrer I, Goutan E, Marin C, Rey MJ, Ribalta T: Brain-derived neurotrophic factor in Huntington disease. Brain Res 2000;866:257-261.

-36 Chauhan NB, Siegel GJ, Lee JM: Depletion of glial cell line-derived neurotrophic factor in substantia nigra neurons of Parkinson's disease brain. J Chem Neuroanat 2001;21:277-288.

37 McBride JL, Ramaswamy S, Gasmi M, Bartus RT, Herzog CD, Brandon EP, Zhou L, Pitzer MR, Berry-Kravis EM, Kordower JH: Viral delivery of glial cell line-derived neurotrophic factor improves behavior and protects striatal neurons in a mouse model of Huntington's disease. Proceedings of the Proc Natl Acad Sci U S A 2006;103:9345-9350.

-38 Allen SJ, Watson JJ, Shoemark DK, Barua NU, Patel NK: GDNF, NGF and BDNF as therapeutic options for neurodegeneration. Pharmacol Ther 2013;138:155-175.

-39 Araujo D. M., D.C. H: Glial cell line-derived neurotrophic factor attenuates the excitotoxin-induced behavioral and neurochemical deficits in a rodent model of Huntington's disease. Neuroscience 1997;81:1099-1110.

40 Alberch J, Perez-Navarro E, Canals JM: Neuroprotection by neurotrophins and GDNF family members in the excitotoxic model of Huntington's disease. Brain Res Bull 2002;57:817-822.

41 Guillemot F, Zimmer C: From cradle to grave: the multiple roles of fibroblast growth factors in neural development. Neuron 2011;71:574-588.

42 Chuang JI, Huang JY, Tsai SJ, Sun HS, Yang SH, Chuang PC, Huang BM, Ching CH: FGF9-induced changes in cellular redox status and HO-1 upregulation are FGFR-dependent and proceed through both ERK and AKT to induce CREB and Nrf2 activation. Free Radic Biol Med 2015;89:274-286.

43 Thisse B, Thisse C: Functions and regulations of fibroblast growth factor signaling during embryonic development. Dev Biol 2005;287:390-402.

-44 Turner N, Grose R: Fibroblast growth factor signalling: from development to cancer. Nat Rev Cancer 2010;10:116-129.

45 Thien CB, Langdon WY: Cbl: many adaptations to regulate protein tyrosine kinases. Nature Rev Mol Cell Biol 2001;2:294-307. 


\section{Cellular Physiology Cell Physiol Biochem 2018;48:605-617 \begin{tabular}{l|l} 
DOI: 10.1159/000491889 & Ond Biochemistry \\
Published online: July 18, 2018 & $\begin{array}{l}\text { 2018 The Author(s). Published by S. Karger AG, Basel } \\
\text { www.karger.com/cpb }\end{array}$
\end{tabular} \\ Yusuf et al.: Neuroprotective Effects of FGF9 in Huntington's Disease}

-46 Apostol BL, Illes K, Pallos J, Bodai L, Wu J, Strand A, Schweitzer ES, Olson JM, Kazantsev A, Marsh JL, Thompson LM: Mutant huntingtin alters MAPK signaling pathways in PC12 and striatal cells: ERK1/2 protects against mutant huntingtin-associated toxicity. Hum Mol Genet 2006;15:273-285.

47 Hasegawa K, Namekawa SH, Saga Y: MEK/ERK signaling directly and indirectly contributes to the cyclical self-renewal of spermatogonial stem cells. Stem cells 2013;31:2517-2527.

48 Sampaio TB, Savall AS, Gutierrez MEZ, Pinton S: Neurotrophic factors in Alzheimer's and Parkinson's diseases: implications for pathogenesis and therapy. Neural Regen Res 2017;12:549-557.

-49 Cheng PH, Li CL, Chang YF, Tsai SJ, Lai YY, Chan AW, Chen CM, Yang SH: miR-196a ameliorates phenotypes of Huntington disease in cell, transgenic mouse, and induced pluripotent stem cell models. Am J Hum Genet 2013;93:306-312.

-50 Hodges A, Strand AD, Aragaki AK, Kuhn A, Sengstag T, Hughes G, Elliston LA, Hartog C, Goldstein DR, Thu D, Hollingsworth ZR, Collin F, Synek B, Holmans PA, Young AB, Wexler NS, Delorenzi M, Kooperberg C, Augood SJ, Faull RL, Olson JM, Jones L, Luthi-Carter R: Regional and cellular gene expression changes in human Huntington's disease brain. Hum Mol Genet 2006;15:965-977. 